\title{
Effects and Influencing Factors of Night Eating Syndrome: A Review of the Literature
}

\author{
Lulu $\mathrm{Li}^{1, *}$ \\ ${ }^{1}$ School of Sanlin, Shanghai, China \\ ${ }^{*}$ Corresponding author. Email: guanghua.ren@gecacademy.cn
}

\begin{abstract}
Obesity is a worldwide public health problem. According to the World Health Organization (WHO), in 2016, the number of overweight adults over 18 years old reached 1.9 billion, of which 650 million were obese. From 1975 to 2016, the global obesity rate has almost tripled. Night Eating Syndrome(NES) is associated with obesity, while it also occurs in nonobese person too. Because NES was defined as higher caloric intake in the evening, the proportion of the syndrome among the world continues increasing and needs more attention. The effects of the syndrome, such as depression, sleep disorders, and weight gain, should not be underestimated. According to the literature, previous research on NES has focused on concepts and therapies such as cognitive behavioural therapies, pharmacological interventions, and light therapies. In addition, the definition was clear, and the results were more positive. The current dominant therapies are pharmacological interventions and CBT therapies. However, the causes of NES and the individual effects and differences are not well studied. Therefore, this paper is a systematic literature review of the above three aspects and summarizes the previous results with international developments. Thus, to provide comprehensive information on NES and to help people learn more about it.
\end{abstract}

Keywords: Night eating syndrome, Eating disorder, Obesity

\section{INTRODUCTION}

\subsection{Night Eating Syndrome Definition}

NES was first introduced by Stunkard et al. [1] to describe a clinical disturbance among a group of obese individuals who were seeking weight loss treatment. They defined the syndrome as a disorder with morning anorexia, insomnia, and consumption of calorie intake more than $25 \%$ after the evening meal. Most patients who suffered from NES reported that their syndromes appeared when their weight gained or life stress increased [1]. Later years, they expanded the definition to include nocturnal ingestions, which were defined as waking up at night to eat [2]. Definitions that do not include time limits also take cultural differences in dinner time into account. For example, most people in countries with a Mediterranean eating style have dinner after $8.30 \mathrm{pm}$ [3]. Once the definition mentioned a specific time, it would be unclear whether dinner was included in the proportion of evening calorie intake. Another research found that calories after 6 p.m., including dinner, account for an average of $63 \%$ of total daily intake. In comparison, calories consumed after 6 p.m., excluding dinner, account for an average of only $33.5 \%$ [4].

In the late 1990s, with the increasing number of obese individuals in America, the attention to NES also increased. The core diagnostic criteria of NES were modified [5]. According to the proposed criteria, the individual must match evening hyperphagia (consumption of calorie intake more than 25\% after the evening meal) and/or two or more nocturnal ingestions per week. Additionally, the individual must experience at least three of the following characteristics: morning anorexia, a strong urge to eat between dinner and sleep and/or during the night, insomnia at least four to five times per week, frequently depress mood during evening hours, belief that eating is necessary to initiate or resume sleep and the awareness during night eating and ability to recall nocturnal ingestions. Also, the syndrome must cause pain and/or functional impairment and could not be better explained by other disorders or external factors. NES is classified in the Diagnostic and Statistical Manual of Mental Disorders (DSM), 5th edition as an "other specified feeding or eating disorder." [6] 


\subsection{The Relationship Between Night Eating Syndrome and Binge Eating}

Binge eating disorder (BED) and NES are two forms of eating disorders associated with obesity. The concept of BED was introduced before NES, but the two share partially similar symptoms in eating disorders. Thus, they are often studied together in comparison. Both prevalences are higher than more traditionally recognized eating disorders, such as bulimia nervosa and anorexia nervosa. Although they occurred in nonobese people, they seem to be associated with long-term weight gain and a higher risk of diabetes and other metabolic dysfunction. They are also associated with a higher risk of psychopathology, including depression, mood, and insomnia, compared to those of similar weight without disorder [5].

According to DSM-5 [5], BED is defined as consuming an unusual amount of food in a short duration and experiencing a loss of control over eating behavior in duration. Additionally, the individual must experience at least three of the following characteristics: consuming food at a much higher speed than normal, continuing eating until uncomfortably full, consuming a large amount of food when not hungry, eating alone to avoid embarrassment, feeling guilty, depressed, or disgusted after eating, having binge eating behavior at least one per week while lasting more than three months on average, a significant amount of distress due to binge episodes. Finally, any regular compensatory behavior must not be accompanied by the disorder, nor should binge episodes occur solely during an episode of bulimia nervosa or anorexia nervosa [6].

NES is more common among people with insomnia, eating disorders and other psychiatric disorders. Also, individuals diagnosed with NES are more likely to have another eating disorder than the general population, and its prevalence is estimated to range from 5\% to $44 \%$ [7]. Almost $15 \%$ to $20 \%$ of patients with NES also have BED. Though there are some repetitive symptoms between BED and NES, it has been suggested that they can be differed by the motivation to eat (for NES is to achieve sleep) and the amount of food eaten per consumption $[8$, 9].

However, the increasing trend of both symptoms should not be underestimated. Therefore, individual and social and cultural differences, physiological and psychological effects and internal and external causes of NES are explained below,

\section{DIFFERENCES}

After a literature survey, it was found that there are differences in NES. There are both external social and cultural differences and internal individual differences. Among the individual differences, there are horizontal gender differences and vertical age differences. Analyzing the differences can help study NES more specifically for different groups and have a more comprehensive understanding of NES.

\subsection{Individual Differences}

\subsubsection{Age Differences}

Through the study of previous literature [10], experimental data suggest age differences in NES. In National Center for Health Statistics [11], elderly people were 2.7 to 3.6 times less likely to present symptoms compared with other age groups. Prevalence is lowest among individuals older than 65 years and highest among young adults ranging from 18 to 30 . In addition, with increasing ages, night eating has become quite general, from a low of $6.5 \%$ in 9-year-old to a high of $21 \%$ in 19 year-old, especially when eating very late in the day. The difference may be because younger adults are more likely to eat late in the day, accounting for the time demands of employment. Meanwhile, individuals over 65 years may no longer work full-time or at all.

\subsubsection{Gender Differences}

The number of studies demonstrated that NES exists gender difference in different groups. In a Swedish population-based twin study, NES was 2.5 times more prevalent in obese men and 2.8 times more prevalent in obese women compared to non-obese men and women [12]. In Nutrition Examination Survey, Men were more likely than women to meet the criteria for night eating [10]. A study about FIT You project toward NES showed that the prevalence of NES was higher in male than female college students [13].

\subsection{Night Eating Syndrome Differences}

\subsubsection{Culture Differences}

As mentioned before, individuals in different cultural groups have different times for dinner. For example, an Italian obesity expert proposed to define NES with relatively late time cutoffs because Americans usually have dinner much earlier than Italians [14]. Having dinner too early or late may both induce NES. Also, the day of the week of night eating matters. It has been generally reported that consuming a considerable proportion of one's daily intake after 7 PM was more often and common on weekends than weekdays. This may reflect on the culture that cooking more abundant dinner or going out to eat with family or friends may mean consuming more than usual. Also, going out to eat has been correlated with eating more than at home $[15,16]$. In China, due to the differences with other countries and the low priority given to NES. A previous study found that though many college students meet the DSM-5 diagnostic criteria for NES, 
nearly one-fifth of the survey respondents tend to nocturnal ingestions. The phenomenon of the night eating among Chinese university students is much higher than that of foreign populations [17].

\subsubsection{Racial Differences}

According to the biobehavioural model [18]. NES exists genetic predisposition. And coupled with eating different customs in each race may lead to Racial Difference. In NHANES-III, black respondents in American were 1.3 to 1.6 times more likely to exhibit night eating than respondents in another racial group [10]. But in other studies, including Caucasian, Hispanic, African American, and Pima Indian ethnic and racial group, the racial difference of NES is not very clear [7]. As previous studies have differed in their conclusions, future experimental studies will need to confirm whether there is a specific interracial difference or a broader racial difference.

\section{INDIVIDUAL EFFECT}

\subsection{Psychological Effect}

NES and BED have similarities in effects. Both are associated with obesity and depression, and anxiety [7]. A previous study demonstrated that NES is more common among individuals seeking weight-loss treatment [1]. Those individuals may suffer low selfesteem and depressed feelings due to unsatisfying their shape but cannot control themselves to eat. Geliebter et al. reported that responders with NES were more likely to have a depressed mood and a higher prevalence of depression [19]. Also, a study of 301 Italian non-clinical adolescents aged 15-19 years showed a significant correlation between NES and depression [20].

There are two possible mechanisms for the association between NES and depression [21]. First, individuals with NES had a significantly higher level of uptaking serotonin transporter than the healthy group. It results in an overall decrease in serotonin within the synapse [22]. And reduced circulating serotonin levels are related to a higher rate of depressive symptoms [23]. Second, NES was accompanied by disturbances in biological rhythms. Haraguchi et al. reported that the eating pattern of nocturnal eating syndrome leads to a phase shift in the peripheral clock, which may cause the development of time-specific depression [24].

Furthermore, some other studies showed that individuals with NES due to morning anorexia and evening hyperphagia felt less hungry though having consumed the amount of intake and had smaller change in hunger afterwards which may represent a general tendency to overeat [25]. Also, individuals with NES mostly do not consider night eating a disorder and do not feel distressed by it [17].

\subsection{Physiological Effect}

While NES is associated with obesity, it may also associate with weight gain. A previous study found that there is no significant difference between the nonobese group and obese group on total or individual scores of night eating questionnaire, except that the obese group with NES were 8/9 years younger than the nonobese group. It demonstrated that NES might be helpful to the development of obesity [26]. The result also fits with the finding that the severity of night eating is associated with high BMI in the middle-aged [27].

In terms of sleep disorders, NES can lead to sleep disorders; however, sleep disorders and insomnia may also predate NES. Studies have found that individuals with NES have difficulty initiating and sustaining sleep [5]. The decrease of nocturnal melatonin levels may worsen sleep disorders, while the increase of corticotropin-releasing factor and cortisol may increase tension and thus stress levels [28].

\section{INFLUENCING FACTORS}

\subsection{External Factors}

\subsubsection{Social Environment}

There are many cultural pressures in today's thinnessas-beauty society. These pressures also take many forms, such as the praise of ultra-thin fashion models and direct messages that one should lose weight. It is often a very negative experience for young people. Overweight adolescents are more likely to be teased. Those who lack social support may pursue an ideal body shape that leads to social acceptance. However, they might be unable to control themselves. Thus NES occurs.

In addition, technological development has accelerated the pace of people's lives and increased stress. In recent years, hot online eating broadcasters have gained attention by eating large amounts of high-calorie food in a short period of time. Multi-camera close-ups of food and the delicate sound of food chewing bring strong sensory stimulation to viewers, triggering their spontaneous perceptual climax [29]. Also, broadcasters usually choose a night to make their lives due to having the largest number of plays. People are also more likely to engage in entertainment activities to relieve stress after a long day of work. This brings misleading information to the audience, giving them the illusion that eating large amounts of food is very stress-relieving and enjoyable. It also stimulates their inner desire for food and induces NES.

Specific comparisons showed that individuals with NES had higher shape, diet, and weight concerns, were more depressed and were more affected by social stress than healthy controls. NES individuals reported higher 
BDI-II [30] and social stress values in the TICS-S [31] compared to the obesity group. Approximately half of the patients with NES showed considerable distress and impairment beyond the clinical significance threshold (moderate distress/impairment). Distress and impairment were particularly associated with the duration of NES. In addition, the analysis showed that distress was significantly correlated with body shape concerns, weight concerns, dietary concerns, and depressive symptoms and social stress. Impairment was significantly associated with social stress [32].

Another important aspect is the food environment in the living area and surrounding community. A previous United States data indicates that $97 \%$ of high schools have vending machines [33]. Another data shows that About $50 \%$ of Canadian schools are situated within $1 \mathrm{~km}$ of a fast-food restaurant, and $60 \%$ are within $1 \mathrm{~km}$ of convenience stores [34]. Large urban and school areas have the most food retailers [35]. Convenience store accessibility is mostly associated with high-calorie food intake, for they typically offer fewer fresh vegetables and fruits.

In addition, with the development of modern technology, take-out and $\mathrm{O} 2 \mathrm{O}$ food delivery systems in some sense reduce people's time for physical activity, such as the walking for dining back and forth, the labor of cooking and washing dishes, thus increasing the risk of chronic diseases such as obesity [36]. It also expands the accessibility of the food environment around the individual, especially making the accessibility of food to the individual during the nighttime period significantly increased, thus inducing night eating.

\subsubsection{Interpersonal Relationships}

There is substantial evidence that comments and teasing about weight are harmful to the mood of overweight children and adolescents. Ridicule about weight is harmful to their mood. Overweight adolescents are at greater risk of being dissatisfied with their bodies [37, 38], experience lower self-esteem [37, 39]. In addition to these negative effects on self-esteem and emotional development, negative comments and teasing about weight and body shape contribute to excessive concern about weight and body shape, thus inducing NES [40].

Further, family criticism would result in long-lasting, negative effects. Johnson et al. reported that people who experienced abuse or neglect during childhood were at greater risk of eating issues [41]. In the study from Taylor et al. [42], $82 \%$ of the sample received negative comments about weight and body shape from family and siblings. Data were analysed to show that regardless of body size, levels of parental criticism were associated with reported emotional abuse and neglect. Higher maximum body size by age 18 was associated with higher levels of emotional neglect. Negative comments from siblings appeared to be more harmful when participants were heavier. In addition, negative comments were higher in families with a higher maximum body size of the biological father. Negative comments were not associated with parental education. Most participants reported only a few negative comments from their parents or siblings. These data indicate that even just a few comments may have a negative impact. In fact, among generally supportive families, a few negative comments may have an incredibly harmful effect because they appear particularly prominent with little or no criticism. The harmful effects of negative comments are confirmed in the data, which suggest that higher emotional abuse scores are associated with poorer self-esteem and lower social support.

\subsubsection{Lack of Healthy Eating Education}

Because education depends on culture, cultural differences are also reflected here. In general, healthy eating education does not attract much social attention yet. Nowadays, children are bombarded with conflicting and confusing messages about their bodies, diet, and weight [43]. Food sellers aim at children and adolescents, tempting them with advertisements describing large portions of unhealthy foods. Meanwhile, social media portray underweight female models as desirable, and male models become muscular and leaner over time [44, 45]. Therefore, the study found that $60 \%$ of girls ranging from 8 years to 11 years have a willingness to lose weight [46] However, another study among 15,349 high school students found that $26 \%$ of female participants had "abnormal diet and weight control" [47]. Also, similar conditions exhibit among males [48]. As mentioned before, the phenomenon of the night eating in China's university student population is much higher than that of foreign populations. But it is lower in medical college students [17]. At present, health education in Chinese universities is mostly focused on mental health education and safety education, and education on healthy eating and regular eating is seldom concerned [49]. This suggests that in the future, universities should add education and publicity on healthy eating and eating routines to the courses of mental health education, to guide college students to establish a healthy diet, improve their health awareness and does not increase unhealthy concerns about restrictive diets or promote negative body image [50].

\subsection{Neuroendocrine Factors}

A previous study [51] indicates that NES has unique neuroendocrine patterns that do not differ between obese and nonobese individuals. Compared to controls, individuals with NES demonstrated attenuation of the normal nocturnal rise in plasma melatonin and leptin levels and overall higher diurnal plasma cortisol levels. 
This may be because low leptin levels lead to a reduction in nocturnal appetite suppression. Since leptin increases less than expected at night, $\mathrm{CRH}$ may increase and contribute to reduced melatonin at night. Low melatonin may lead to nocturnal sleep disruption. These neuroendocrine findings may explain the unexpectedly high rate of the night eating in NES subjects [52].

The study [51] also reported a high carbohydrate to protein ratio during seizures $(7: 1)$. It is hypothesized that patients may restore disrupted sleep by eating highcarbohydrate food on awakening, thereby increasing the supply of tryptophan to the brain and conversion to serotonin [52].

In terms of glycaemic control and heritability, another research shows that the prevalence of NES is $9.7 \%$ in the patient with diabetes [53]. Night eating is also associated with poorer glycaemic control, which may be a result of the food choices made by those who eat at night, including high carbohydrate and high-fat foods [54]. And in a study comparing families who had night eating behaviors with those who did not, first-degree relatives of individuals who had night eating behaviors had a greater probability of having the disorder than those in the control group, suggesting a heritability [55].

In 1955, Stunkard and his colleagues proposed that significant life stress worsened night eating symptoms and removing a person from a stressful environment would reduce the symptoms [1]. Later research has found that stress, and mental illnesses such as depression, are often associated with the Initial onset and maintenance of night eating [56]. Social stress has proven to induce a strong cortisol response [57]. Cortisol is a hormone released by the adrenal cortex in response to stress and is correlated with overeating and weight gain [58]. No differences in serum cortisol levels were found between obese and non-obese individuals [51]. Therefore, there exists the hypothesis that night eating may be related to impaired regulation of the hypothalamic-pituitaryadrenal axis that controls the cortisol stress response [59].

\section{CONCLUSION}

NES is both a social difficulty and a social hotspot, while its increasing trend has gradually become significant in recent years, so the study of NES is beneficial to society and individuals and has certain practical significance. NES exists differences in ages, genders, culture, and race. It also causes effects both on psychology and physiology like obesity, depression, and sleep disorders. The social environment can influence it, interpersonal relationship, and neuroendocrine factors. Since the research on NES is not yet comprehensive, the related research in some factors is still in the initial stage. There are certain limitations in this study due to the authors' time and ability level. Future scholars who studied in NES can conduct long-period experiments to analyze the differences in NES and the reasons for the differences, which may help to have a deeper level of recognition of NES and thus improve the existing treatment methods.

\section{REFERENCES}

[1] Stunkard, A.J., W.J. Grace \& H.G. Wolff. 1955. The night- eating syndrome: a pattern of food intake among certain obese patients. Am. J. Med. 19: 7886.

[2] Rand, C. S., Macgregor, A. M., \& Stunkard, A. J. (1997). The night eating syndrome in the general population and among postoperative obesity surgery patients. International Journal of Eating Disorders, 22(1), 65-69.

[3] Adami, G. F., Meneghelli, A., \& Scopinaro, N. (1997). Night eating syndrome in individuals with Mediterranean eating style. Journal of Eating and Weight Disorders, 2, 203-206.

[4] Allison, K. C., O’Reardon, J., Stunkard, A. J., \& Dinges, D. (2001). Characterizing the night eating syndrome. Obesity Research, 9, 93S.

[5] McCuen-Wurst, C., Ruggieri, M., \& Allison, K. C. (2018). Disordered eating and obesity: associations between binge eating-disorder, night-eating syndrome, and weight-related comorbidities. Annals of the New York Academy of Sciences, 1411(1), 96.

[6] Edition, F. (2013). Diagnostic and statistical manual of mental disorders. Am Psychiatric Assoc, 21.

[7] Vander Wal, J.S. 2012. Night eating syndrome: a critical review of the literature. Clin. Psychol. Rev. 32: 49-59.

[8] de Zwaan, M., M. Marschollek \& K.C. Allison. 2015. The night eating syndrome (NES) in bariatric surgery patients. Eur. Eat. Disord. Rev. 23: 426-434.

[9] de Zwaan, M., A. Muller, K.C. Allison, et al. 2014. Prevalence and correlates of night eating in the German general population. PLoS One 9: e97667.

[10] Striegel - Moore, R. H., Franko, D. L., Thompson, D., Affenito, S., \& Kraemer, H. C. (2006). Night eating: prevalence and demographic correlates. Obesity, 14(1), 139-147.

[11] National Center for Health Statistics. Plan and operation of the Third National Health and Nutrition Examination Survey, 1988-94. Series 1: programs and collection procedures. Vital Health Stat 1. 1994;Jul:1-407.

[12] Tholin, S., A. Lindroos, P. Tynelius, et al. 2009. Prevalence of night eating in obese and nonobese twins. Obesity 17: 1050-1055. 
[13] Guo, F., Tian, Y., Cui, Y., \& Huang, C. (2020). Night-eating syndrome and depressive symptoms in college freshmen: fitness improvement tactics in youths (FITYou) project. Psychology research and behavior management, 13, 185.

[14] Adami GF, Meneghelli A, Scopinaro N. Night eating and binge eating disorder in obese patients. Int J Eat Disord. 1999;25:335-8.

[15] Kant AK, Graubard BI. Eating out in America, 1987-2000: trends and nutritional correlates. Prev Med. 2004;38:243-9.

[16] Thompson OM, Ballew C, Resnicow K, et al. Food pur- chased away from home as a predictor of change in BMI z-score among girls. Int J Obes Relat Metab Disord. 2004;28: 282-9.

[17] ZHENG Ya-nan, HU We, GONG Xi, ZHAO Ameng. Current situation and influencing factors of the night eating phenomenon among college students Modern Preventive Medicine, 2018, Vol. 45, NO. 3.

[18] Stunkard, A. J., Allison, K. C., Lundgren, J. D., \& O'Reardon, J. P. (2009). A biobehavioural model of the night eating syndrome. Obesity Reviews, 10(Suppl. 2), 69-77.

[19] Geliebter, A., McOuatt, H., Tetreault, C. B., Kordunova, D., Rice, K., Zammit, G., \& Gluck, M. (2016). Is night eating syndrome associated with obstructive sleep apnea, BMI, and depressed mood in patients from a sleep laboratory study?. Eating behaviors, 23, 115-119.

[20] Riccobono G, Pompili A, Iannitelli A, Pacitti F. The relationship between night eating syndrome, depression and chronotype in a non-clinical adolescent population. Riv Psichiatr. 2019;54(3):11 5-119.

[21] Guo, F., Tian, Y., Cui, Y., \& Huang, C. (2020). Night-eating syndrome and depressive symptoms in college freshmen: fitness improvement tactics in youths (FITYou) project. Psychology research and behavior management, 13, 185.

[22] Lundgren JD, Newberg AB, Allison KC, Wintering NA, Ploessl K, Stunkard AJ. I-121-ADAM SPECT imaging of serotonin transporter binding in patients with night eating syndrome: a preliminary report. Psychiatry Res. 2008;162(3):214-220.

[23] Payne JK, Piper BF, Rabinowitz I, Zimmerman MB. Biomarkers, fatigue, sleep, and depressive symptoms in women with breast can- cer: a pilot study. Oncol Nurs Forum. 2006;33(4):775-783.
[24] Haraguchi A, Fukuzawa M, Iwami S, et al. Night eating model shows time-specific depression-like behavior in the forced swimming test. Sci Rep. 2018;8(1):1-4.

[25] GluckME, GeliebterA, SatovT. Night eating syndrome is associated with depression, low self esteem, reduced daytime hunger, and less weight loss in obese outpatients[J]. Obesity Research, 2001, 9(4) : 264 -267.

[26] Marshall, H.M., K.C. Allison, J.P. O’Reardon, et al. 2004. Night eating syndrome among nonobese persons. Int. J. Eat. Disord. 35: 217-222.

[27] Meule, A., K.C. Allison, E. Brahler, et al. 2014. The association between night eating and body mass depends on age. Eat. Behav. 15: 683-685.

[28] Hazlerigg, D. G. (2001). What is the role of melatonin within the anterior pituitary? The Journal of Endocrinology, 170(3), 493-501.

[29] Shi Yuying. Audience aesthetics and consumption alienation of online eating broadcast under the landscape society--Eating broadcast on BiliBili website as an example[J]. Yiyuan,2019(04):75-78.

[30]Ku hner,C.,Burger,C.,Keller,F.,Hautzinger,M.,2007. Reliabilityandvalidityofthe revised Beck Depression Inventory (BDI-II). Results from German samples. Nervenarzt 78 (6), 651-656.

[31] Schulz, P., Schlotz, W., Becker, P., 2004. Das Trierer Inventar zum chronischen Stress-Manual. Hogrefe, Go ttingen.

[32] Fischer S, Meyer AH, Hermann E, et al. Night eating syndrome in young adults: Delineation from other eating disorders and clinical significance[J]. Psychiatry Research, 2012, 200( 2/3) : 494 - 501.

[33] Finkelstein DM, Hill EL, Whitaker RC. School food environments and policies in US public schools. Pediatrics. 2008;122:e251-9.

[34] Seliske LM, Pickett W, Boyce WF, Janssen I. Density and type of food retailers surrounding Canadian schools: variations across socioeconomic status. Health Place. 2009;15:903-7.

[35] Browning, H. F., Laxer, R. E., \& Janssen, I. (2013). Food and eating environments: in Canadian schools. Canadian journal of dietetic practice and research, 74(4), 160-166.

[36] Mayila Maimaiti. The development of a food environment perception questionnaire and a preliminary analysis on association between food environment and obesity [D]. Zhejiang University, 2019 
[37] Grilo CM, Wilfley DE, Brownell KD, Rodin J. Teasing, body image, and self-esteem in a clinical sample of obese women. Addict Behav. 1994; 19:443-450

[38] Vander Wal JS, Thelen MH. Predictors of body image dissatisfaction in elementary-age school girls. Eat Behav. 2000;1: 105-122.

[39] Pesa JA, Syre TR, Jones E. Psychosocial differences associated with body weight among female adolescents: the importance of body image. J Adolesc Health. 2000; 26:330 -337

[40] Jacobi C, Hayward C, de Zwaan M, Kraemer HC, Agras WS. Coming to terms with risk factors for eating disorders: application of risk terminology and suggestions for a general taxonomy. Psych Bull. 2004; 130:19-65.

[41] Johnson JG, Cohen P, Kasen S, Brook JS. Childhood adversi- ties associated with risk for eating disorders or weight problems during adolescence or early adulthood. Am J Psychiatry. 2002; 159:394- 400

[42] Taylor, C. B., Bryson, S., Doyle, A. A. C., et al (2006). The adverse effect of negative comments about weight and shape from family and siblings on women at high risk for eating disorders. Pediatrics, 18(2), 731-738.

[43] Cook-Cottone, C. P., Tribole, E., \& Tylka, T. L. (2013). Healthy eating in schools: Evidence-based interventions to help kids thrive. American Psychological Association.

[44] Hawks, S.R., Merrill, R.M., Madanat, H.N., Miyagawa, T., Suwanteerangkul, J., Guarin, C.M. and Shaofang, C. (2004), "Intuitive eating and the nutrition transition in Asia", Asia Pacific Jounral of Clinical Nutrition, Vol. 13 No. 2, pp. 194-203.

[45] Buote, V.M., Wilson, A.E., Strahan, E.J., Gazzola, S.B. and Papps, F. (2011), "Setting the bar: divergent sociocultural norms for women's and men's ideal appearance in real-world contexts", Body Image, Vol. 8 No. 4, pp. 322-334.

[46] Phares, V., Steinberg, A.R. and Thompson, J.K. (2004), "Gender differences in peer and parental influences: body image disturbance, self-worth, and psychological functioning in preadolescent children", Journal of Youth and Adolescence, Vol. 33 No. 5, pp. 421-429.

[47] Forman-Hoffman, V. (2004), "High prevalence of abnormal eating and weight control practices among US high-school students", Eating Behavior, Vol. 5 No. 4, pp. 325-336.
[48] Grogan, S. and Richards, H. (2002), "Body image: focus groups with boys and men", Men and Maculinities, Vol. 4 No. 3, pp. 219-232.

[49] Browning, H. F., Laxer, R. E., \& Janssen, I. (2013). Food and eating environments: in Canadian schools. Canadian journal of dietetic practice and research, 74(4), 160-166.

[50] LIU Chang-zheng, LEI Bo. Survey on requirements of healthy diet education in comprehensive university. Modern Preventive Medicine, 2013, Vol.40, NO.13.

[51] Birketvedt, G., Sundsfjord, J., Sundsfjord, J., Osterud, B., Dinges, D., Bilker, W., \& Stunkard, A. (1999). Behavioral and neuroendocrine characteristics of the night eating syndrome. Journal of the American Medical Association, 282, 657-663.

[52] De Zwaan, M., Burgard, M. A., Schenck, C. H., \& Mitchell, J. E. (2003). Night time eating: a review of the literature. European Eating Disorders Review: The Professional Journal of the Eating Disorders Association, 11(1), 7-24.

[53] Morse, S.A., P.S. Ciechanowski, W.J. Katon, et al. 2006. Isn't this just bedtime snacking? The potential adverse effects of night-eating symptoms on treatment adherence and out- comes in patients with diabetes. Diabetes Care 29: 1800- 1804.

[54]Hood,M.M.,S.Reutrakul\&S.J.Crowley.2014.Nightea ting in patients with type 2 diabetes. Associations with glycemic control, eating patterns, sleep, and mood. Appetite 79: 91-96.

[55] Allison, K.C., J.D. Lundgren, J.P. O'reardon, et al. 2010. Pro- posed diagnostic criteria for night eating syndrome. Int. J. Eat. Disord. 43: 241-247.

[56] Marchesini, G., S. Calugi, R. Marzocchi, et al. 2013. Night eating syndrome in obesity. In Handbook of Nutrition, Diet and Sleep. V.R. Preedy, V.B. Patel \& L.-A. Le, Eds.: 104-120. Wageningen: Wageningen Academic Publishers.

[57] Vander Wal, J. S., Maraldo, T. M., Vercellone, A. C., \& Gagne, D. A. (2015). Education, progressive muscle relaxation therapy, and exercise for the treatment of night eating syndrome. A pilot study. Appetite, 89, 136-144.

[58] Epel, E., R. Lapidus, B. McEwen, et al. 2001. Stress may add bite to appetite in women: a laboratory study of stress-induced cortisol and eating behavior. Psych neuroendocrinology 26: 37-49.

[59] Ungredda, T., M.E. Gluck \& A. Geliebter. 2012. Pathophys- iological and neuroendocrine aspects of night eating syn- drome. In Night Eating Syndrome: 
Research, Assessment, and Treatment. J.D. Lundgren, K.C. Allison \& A.J. Stunkard, Eds.: 197217. New York: Guilford. 